\title{
On Excited Meson Spectra in the Scalar Strong Interaction Hadron Theory
}

\author{
F. C. Hoh \\ Retired, Dragarbrunnsg. 55C, 75320 Uppsala, Sweden \\ Email: hoh@telia.com
}

How to cite this paper: Hoh, F.C. (2017) On Excited Meson Spectra in the Scalar Strong Interaction Hadron Theory. Journal of Modern Physics, 8, 1127-1133. https://doi.org/10.4236/jmp.2017.88073

Received: June 3, 2017

Accepted: June 25, 2017

Published: June 28, 2017

Copyright $\odot 2017$ by author and Scientific Research Publishing Inc. This work is licensed under the Creative Commons Attribution International License (CC BY 4.0).

http://creativecommons.org/licenses/by/4.0/

\begin{abstract}
Meson spectra have been treated earlier in the scalar strong interaction hadron theory, choosing the Coulomb and linear type of potentials, neglecting the quadratic one. The spectra of ground state pseudoscalar and vector mesons were adequately accounted for but not that of the excited mesons. Here, the quadratic potential replaces the Coulomb one and the same ground state meson spectra were recovered. Also, the masses of low-lying radially excited pseudoscalar and vector mesons were found to be $4 \%-18 \%$ smaller than the measured ones. Here, the linear type of potential, by itself of nonlinear nature, has been neglected. For some orbitally excited pseudoscalar mesons, the difference is $14 \%-38 \%$. The discrepancies are tentatively attributed to the neglected nonlinear potential, which is expected to increase with meson mass, as can be seen in the tables below.
\end{abstract}

\section{Keywords}

Excited Meson Spectra, Quadratic Confinement, Scalar Strong Interaction

\section{Introduction}

The Schrödinger-Dirac equations became an established theory because of their ability to account for atomic spectra in the early stages of development. Similarly, any viable hadron theory must be able to account at least approximately for the meson spectra. Quantum Chromodynamics (QCD) (see e.g. [1]), the main stream strong interaction theory, has failed to do this, after decades of work and lattice computations. Therefore, the low energy, nonperturbative end of QCD has to be abandoned.

On the other hand, the scalar strong interaction hadron theory (SSI) can approximately but adequately account for the masses of the ground state pseudoscalar mesons $\sigma^{-}$(singlet) and vector mesons $I^{-}$(triplet) [2] [3] [4]. However, 
predictions of the spectra of the excited states of these mesons using the same linearized equations turned out to contradict data [5]; the spacing between the energy levels according to (7) below turned out to be too small. The nonlinear strong interaction potential was called in to mitigate this difficulty phenomenologically ([4] Section 5.5-7).

This difficulty is incompatible with a viable SSI. The purpose of this paper is to resolve it and provide predictions in rough agreement with data without the above phenomenology.

\section{Background, Coulomb and Linear Type of Potential}

In SSI, the interaction potential between the quark and the antiquark in a meson is given in ([2] 7.2, [4] 3.2.8),

$$
\begin{gathered}
\Phi_{m}(\underline{x})=-\Phi_{c}(\underline{x})+\frac{d_{m}}{r}+d_{m 0}+d_{m 2} r^{2} \\
\Phi_{c}(\underline{x})=\frac{g_{s}^{4}}{8 \pi} \int \mathrm{d}^{3} \underline{x}^{\prime}\left|\underline{x}-\underline{x}^{\prime}\right| \operatorname{Re}\left(\underline{\psi}\left(\underline{x}^{\prime}\right) \underline{\chi^{*}}\left(\underline{x}^{\prime}\right)-\psi_{0}\left(\underline{x}^{\prime}\right) \chi_{0}^{*}\left(\underline{x}^{\prime}\right)\right)
\end{gathered}
$$

Here, $\underline{x}$ is the interquark distance vector and also denotes the "hidden" relative space, $r=|\underline{x}|$, the $d_{m}$ 's integration constants of the fourth order differential equation ([2] 6.9, [4] 3.1.11), $g_{s}$ the strong interaction coupling constant, $\psi_{o}$ (singlet) and $\psi$ (triplet) the rest frame meson wave functions in $\underline{x}$. In the case of zero orbital momentum, $l=0$, these wave functions are determined by ([4] 3.2.5b, 3.4.1, 3.4.2a, 3.4.3)

$$
\begin{gathered}
\psi_{0}(\underline{x})=\psi_{0}(r), \quad \underline{\psi}(\underline{x})=\hat{r} \psi_{1}(r), \quad \hat{r}=\underline{x} / r \\
\left(\frac{1}{r^{2}} \frac{\partial}{\partial r} r^{2} \frac{\partial}{\partial r}-\frac{J(J+1)}{r^{2}}-\Phi_{c J}(r)+\frac{d_{m}}{r}+d_{m 0}+d_{m 2} r^{2}+\frac{E_{J}^{2}-\left(m_{p}+m_{r}\right)^{2}}{4}\right) \psi_{J}(r)=0
\end{gathered}
$$

derived from ([4] 3.2.10b, 3.2.11a). Here $m_{p, r}$ are quark masses of flavors $p, r, J=$ 0 refers to singlet and $J=1$ to triplet and (2) becomes ([4] 3.2.17)

$$
\Phi_{c J}(r)=\frac{g_{s}^{4}}{6}\left[\int_{0}^{r} \mathrm{~d} r^{\prime} r^{\prime 2}\left|\psi_{J}\left(r^{\prime}\right)\right|^{2}\left(3 r+\frac{r^{\prime 2}}{r}\right)+\int_{r}^{\infty} \mathrm{d} r^{\prime} r^{\prime}\left|\psi_{J}\left(r^{\prime}\right)\right|^{2}\left(3 r^{\prime 2}+r^{2}\right)\right]
$$

In early 1990's, when the above work was in progress, potential models suggested a confinement potential of the Coulomb plus linear type ([6], [7] \$14.3.2). The nonlinear $\Phi_{c J}$ leads to linear confinement at large $r$ ([2] 7.11b [4] 3.2.19). The Coulomb term $d_{m} / r$ was kept and the quadratic term $d_{m 2} r^{2}$ dropped ([4] 3.2.20). In this case, the linearized (4) with $\Phi_{c \zeta} \rightarrow 0$ is of the same form as that for the hydrogen atom and the ground state solutions are given by ([4] 4.3.1-3)

$$
\psi_{00}(r)=\sqrt{\frac{d_{m}^{3}}{8 \pi \Omega}} \exp \left(-d_{m} r / 2\right), \quad \psi_{10}(r)=\sqrt{\frac{d_{m}^{5}}{3072 \pi \Omega}} r \exp \left(-d_{m} r / 4\right)
$$

where the second subscript refers to radial quantum number $n_{r}=0$. These wave functions, being plane waves in the laboratory frame $\underline{X}$, vanish when the normalization volume $\Omega \rightarrow \infty$ ([4] 4.7.2) so the above assumption $\Phi_{c J} \rightarrow 0$ holds. The meson mass $E_{J 0}$ is given by a slightly extended ([4] 4.4.1) 


$$
E_{J n}^{2}=\left(m_{p}+m_{r}\right)^{2}-4 d_{m 0}-\left(d_{m} /\left(n_{r}+J+1\right)\right)^{2}
$$

This result with $n_{r}=0$ and the empirical $E_{10}^{2}-E_{00}^{2} \approx 0.56 \mathrm{GeV}^{2} \quad$ ([4] 5.2.2) together with six pseudoscalar meson masses determine the five quark masses, $d_{m}$, and $d_{m o}$ ([2] 10.2, [3] Table 1, [4] 5.2.3, Table 5.1). The results are summarized in Table 1 below.

Table 1 and (7) predict many other ground state pseudoscalar and vector meson masses with good approximation ([3], [4] 5.2.3, Tables 5.3-5).

However, for radially excited pseudoscalar and vector mesons, $n_{r} \geq 1$ and (7) shows that the spacings between successive radially excited meson masses, analogous to those between excited states in a hydrogen atom, are too small and are decreasing rapidly with increasing $n_{r}$, contrary to data ([4] Tables 5.6-7). In an attempt to remove this discrepancy, an assumption ([4] 4.7.5) was made in which the normalization volume $\Omega \rightarrow \Omega_{c}$ is made finite in (6) for the excited states. The wave functions in the nonlinear $\Phi_{c J}$ in (5) now no longer vanish. Since $\Phi_{c J}$ is a positive quantity, it will increase the meson masses.

Not being able to treat this complex nonlinear problem, $\Phi_{m}(r)$ in $(1)$ was replaced by unknown parameters ([4] 5.5.2) which are determined by using data points, the masses of chosen excited mesons. In this way, the results in ([4] Tables 5.6-7) were obtained, after having spent many data points.

Obviously, the above treatment failed to account for the spectra of excited mesons.

\section{Quadratic Confinement}

In reviewing the above treatment, it is seen that there is no compelling justification to drop $d_{m 2} r^{2}$ in (1), (4), as was done below (5), except that the linearized (4) with $\Phi_{c J}=0$ turns out to have no converging solution. Therefore, put $d_{m}=0$ and keep the quadratic confining $d_{m 2} r^{2}$ in (4). The solution analogous to (6) is of harmonic oscillator type and reads

$$
\begin{gathered}
\psi_{J n}(r)=\frac{1}{\sqrt{\Omega}} \exp \left(-\frac{d_{h}}{2} r^{2}\right) \sum_{m=0}^{n} a_{m} r^{s+m}, \\
d_{m 2}=-d_{h}^{2}, d_{h}, a_{0}>0, a_{\text {odd }}=0, n=2 n_{r} \\
\psi_{J 0}(r)=\frac{1}{\sqrt{\Omega}}\left(\frac{d_{h}}{\pi}\right)^{3 / 4} \exp \left(-\frac{d_{h}}{2} r^{2}\right)
\end{gathered}
$$

where $\Omega$ denotes the nomalization box in ([4] 4.2.8) for the ground state ( $8 b)$.

The series in (8a) terminates when

$$
\begin{aligned}
& a_{n+2}=\frac{2 d_{h}\left(s+n+\frac{3}{2}\right)-\frac{1}{4}\left(E_{J}^{2}-\left(m_{p}+m_{r}\right)^{2}\right)-d_{m 0}}{(s+n+2)(s+n+3)-J(J+1)} a_{n}=0, \\
& s(s+1)=J(J+1), s=J
\end{aligned}
$$

Table 1. Quarks masses and two integration constants in SSI obatined in [3, 2]. These are also reproduced in ([4] 5.2.3 and Table 5.1).

\begin{tabular}{ccccccc}
\hline$m_{u}(\mathrm{Gev})$ & $m_{d}-m_{u}$ & $m_{s}$ & $m_{c}$ & $m_{b}$ & $d_{m 00}\left(\mathrm{GeV}^{2}\right)$ & $d_{m}(\mathrm{GeV})$ \\
\hline 0.6592 & 0.00215 & 0.7431 & 1.6215 & 4.7786 & 0.24455 & $\approx 0.864$ \\
\hline
\end{tabular}


Here, the other root $s=-J-1$ leads to divergent wave function at $r=0$ and is dropped. Now, (7) is changed to

$$
\frac{1}{4} E_{J n}^{2}=\frac{1}{4}\left(m_{p}+m_{r}\right)^{2}-d_{m 0}+2 d_{h}\left(s+n+\frac{3}{2}\right), \quad s=J
$$

which differs from (7) only in the last term. The above ground state results for $n_{r}$ $=0$ mentioned in Table 1 and the two lines below it can now be taken over if the last two columns in Table 1 are replaced by

$$
d_{m 0}=0.641126 \mathrm{GeV}^{2}, \quad d_{h}=0.07 \mathrm{GeV}^{2}
$$

\section{Radially Excited Mesons}

Comparison of (7) to (10) shows that the latter gives much larger spacings between the excited states. Application of (10) to the radially excited states in ([4] Table 5.6, 5.7) are given in Table 2 and Table 3 below.

Table 2. Masses (MeV) of low-lying excited singlet $l=0$ mesons considered in ([4] Table 5.6). Data $E_{\text {exp }}$ [5] are given in brackets [..]. Below these are the predicted masses $E_{t h}$ from (10) using Table 1. $N=n_{r}+1$. The differences $\Delta E^{2} / 4=\left(E_{\text {exp }}^{2}-E_{t h}^{2}\right) / 4$ are shown in parentheses (..). The mass term fior $\eta$ in (7) is given by ([4] 2.4.15). $\eta_{c}$ is a $\bar{c} c$ state.

\begin{tabular}{cccc}
\hline Isospin & $N^{2 S+1} I_{J}=1^{1} S_{0}$ & $=2^{1} S_{0}$ & $=3^{1} S_{0}$ \\
\hline 1 & $\pi$ & $\pi(1300)[1200-1400]$ & $\pi(1800)[1812 \pm 13]$ \\
& & $1067.2(0.1378)$ & $1502(0.2568)$ \\
$1 / 2$ & $K$ & $K(1460)[1400,1460]$ & $K(1830)[\approx 1830]$ \\
& & $1166.9(0.1496,0.1925)$ & $1575.2(0.2169)$ \\
0 & $\eta$ & $\eta(1295)[1294 \pm 4]$ & $\eta(1760)[1760 \pm 11]$ \\
& & $1204(0.0562)$ & $1574(0.155)$ \\
0 & $\eta_{c}$ & $\eta_{c}(2 S)[3638 \pm 5]$ & \\
& & $3148.3(0.8308)$ & \\
\end{tabular}

Table 3. Masses $(\mathrm{MeV})$ of low-lying radially excited triplet $l=0$ mesons considered in ([4] Table 5.7). Data $E_{\text {exp }}$ [5] are given in brackets [..]. Below these are the predicted masses $E_{t h}$ from (10) using Table 1. $N=n_{r}+1$. The differences $\Delta E^{2} / 4=\left(E_{\text {exp }}^{2}-E_{t h}^{2}\right) / 4$ are shown in parentheses (..).

\begin{tabular}{ccc}
\hline Isospin & $N^{2 S+1} I_{J}=1^{3} S_{0}$ & $=2^{3} S_{1}$ \\
\hline 1 & $\rho$ & $\rho(1450)[1465 \pm 25]$ \\
& & $1303.3(0.1119)$ \\
0 & $\omega$ & $\omega(1420)[1400-1450]$ \\
$1 / 2$ & $K^{*}(892)$ & $1303.3(0.06535-0.101)$ \\
& & $K^{*}(1410)[1414 \pm 15]$ \\
0 & $\phi$ & $1166.8(0.1595)$ \\
0 & $J / \psi$ & $\phi(1680)[1680 \pm 20]$ \\
& & $1471(0.1646)$ \\
0 & $Y(1 S)$ & $Y(2 S)[10023.26 \pm 0.1 \pm 0.084]$ \\
& & $9555(2.2919)$ \\
\end{tabular}


The predicted masses $E_{t h}$ are $7 \%$ - $18 \%$ smaller than the measured ones $E_{\text {exp }}$ in Table 2 and $4 \%-18 \%$ in Table 3. These differences may tentatively be attributed to the neglected nonlinear potential $\Phi_{c \gamma}(r)$ of (5) in (4) in order to arrive at (10). Actually, (4) and (5) have been solved numerically using an iterative procedure. ([2] Section 10, [3] Section 7), also explained in ([4] Section 5.6), for the first and second radially excited singlet and triplet mesons in Section $2\left(d_{m 2}=0\right)$. The wave functions as well as the associated nonlinear potential $\Phi_{c \gamma}(r)$ are plotted in ([2] Figure 1 and Figure 2, [3] Figure 2 and Figure 3). These computations depend upon the choices of unknown parameters, the amplitudes of the wave function in $\Phi_{c}$ in (2) or the finite sizes of the normalization box $\Omega_{c}$ of ([4] 4.7.5a) or, equivalently, $N_{C J n}$ of ([3] $4 \mathrm{~d}$ ) which is a volume integral over $\psi_{J}(r)^{2}$. Therefore, the contribution of $\Phi_{c \gamma}(r)$ to $E_{t h}$ can presently not be uniquely determined. More strictly, an $\underline{X}$ dependence is implicitly introduced in (4.7.4a) so that the general wave function $\psi_{j}(X, X)$ of ([4] 3.1.5) is no longer separable in the laboratory coordinates $\left(X_{0}, \underline{X}\right)$ and relative coordinates $\left(x_{0}, \underline{x}\right)$, rendering the problem not manageable.

Qualitatively, follow Section 5.5 of [4] and let $\Phi_{c)}(r)$ in (4) be replaced by constants $\bar{\Phi}_{c J}$. Correct predictions are achieved if $\bar{\Phi}_{c J}=\Delta E^{2} / 4=\left(E_{\text {exp }}^{2}-E_{t h}^{2}\right) / 4$ shown in the parentheses ( ) in Table 2 and Table 3. If the nonlinear potential contribution is small relative to the quadratic confining potential, $\bar{\Phi}_{c J}<<$ the last term in (10). Then (8a) and hence also $\Phi_{c l}(r)$ of (5) still hold approximately; $\bar{\Phi}_{c J}$ becomes independent of flavor or quark masses and be a constant in each column of Table 2 and Table 3 . The values $\Delta E^{2} / 4$ in the parentheses are however not constant in each column. This due to that they are not small but comparable to the last term in $(10)=3 d_{h}, 5 d_{h}, 7 d_{h} \ldots=0.21,0.35,0.49 \ldots$ Therefore, $\bar{\Phi}_{c J}$ is flavor-dependent and this dependence, as well as that for $\psi_{f}$, increase with decreasing normalization volume $\Omega \rightarrow \Omega_{c}$, which generally decrease with increasing mass. These are seen from the numbers in the parenthese (..) in Table 2 and Table 3 in which $\Delta E^{2} / 4$ become large for heavier mesons. Thus, the nonlinear potential $\Phi_{c J}$ does contribute to the meson masses, in this case by $9-18 \%$ mentioned above.

\section{Orbitally Excited Singlet Mesons}

The wave function is given by ([4] 3.4.2a), $\psi_{0}(\underline{x})=\psi_{0 l}(\underline{x}) \Psi_{l m}(\theta, \varphi)$, where $\theta$ and $\varphi$ are angles and $l \geq 1 . \Phi_{c J}(\underline{x})$ in (2) now depend upon these angles and (5) and hence also (4) no longer hold. For $r \rightarrow 0$, the wave function (8a) $\propto r^{s}$ which vanishes for $s=1 \geq 1$ and is therefore independent of the angles. For large $r$, the $r^{2}$ term in (1) dominates over $\Phi_{c}(\underline{x})$ of (2) which by itself is proportional to $r$ ([4] 3.2.19). In these both limits. the equivalent of ([4] 3.4.2b) analogous to (4) reads

$$
\left(\frac{1}{r^{2}} \frac{\partial}{\partial r} r^{2} \frac{\partial}{\partial r}-\frac{l(l+1)}{r^{2}}-\Phi_{c 0}\left(\begin{array}{l}
r \rightarrow 0 \\
r \rightarrow \infty
\end{array}\right)+d_{m 0}-d_{h}^{2} r^{2}+\frac{E_{0 l}^{2}-\left(m_{p}+m_{r}\right)^{2}}{4}\right) \psi_{0 l}(r)=0
$$


As was mentioned in ([4] \$5.7.1), the two solutions for small and large $r$ determined from (12) are independent of the angle $\theta$ and are to be connected by an unknown solution dependent upon both $r$ and $\theta$ in the intermediate $r$ region. This nonseparable problem starting from ([4] 3.2.10b) is, as in [4] $\$ 5.7 .1$, beyond the reach of the present work.

Not being to treat this problem adequately, follow the procedures that led to Table 2 and Table 3 in order to obtain some estimates. Linearizing (12) by putting $\Phi_{c 0}=0$, (10) with $n=0$ and $s=l$ is obtained. It gives the masses of the mesons in Table 5.8 of [4] listed in Table 4 below.

These results are not surprisingly coarser than those in Table 2 and Table 3 because the neglected nonlinear $\Phi_{c 0}$ in (12) actually contains angle dependence in the intermediate $r$ region; more equations are required. This turns out actually to be the case; the predicted masses $E_{t h}$ are now $14 \%$ - 38\% smaller than the measured $E_{\text {exp }}$, as compared to $9 \%$ - $18 \%$ for the radially excited mesons. Again replace $\Phi_{c 0}$ in (12) by constants $\bar{\Phi}_{c 0}$ and put it equal to $\Delta E^{2} / 4=\left(E_{\text {exp }}^{2}-E_{\text {th }}^{2}\right) / 4$ shown in the parentheses (..) in Table 4 . The values in them are now much greater than the last term in $(10)=3 d_{h}, 5 d_{h}, 7 d_{h} \ldots=0.21$, $0.35,0.49 \ldots$, showing that the nonlinear potential $\Phi_{c o}$ contributes much more to the masses than does the quadratic confining potential.

Like the behavior mentioned at the end of Section $4, \Delta E^{2} / 4$ here also increase with the meson masses and, in addition, also with increasing orbital angular momentum $l$.

Table 4. Masses (MeV) of low-lying orbitally excited $l \geq 1$ singlet mesons in ([4] Table 5.8). Data $E_{\text {exp }}$ [5] are given in brackets [..]. Below these are the predicted masses $E_{t h}$ from (10) with $n=0$ and $s=1$ using Table 1 . The differences $\Delta E^{2} / 4=\left(E_{\text {exp }}^{2}-E_{\text {th }}^{2}\right) / 4$ are shown in parentheses (..). * in front denotes a state not present in the quark model assignments in [5].

\begin{tabular}{|c|c|c|c|c|}
\hline Quark-content & $\begin{array}{c}N_{r}^{2 S+1} I_{J}=1^{1} P_{1} \\
J^{P}=1^{+}\end{array}$ & $\begin{array}{c}=1^{1} D_{2} \\
=2^{-}\end{array}$ & $\begin{array}{c}=1^{1} F_{3} \\
=3^{+}\end{array}$ & $\begin{array}{c}=1^{1} G_{4} \\
=4^{-}\end{array}$ \\
\hline $\bar{d} u, \bar{d} d, \bar{u} u$ & $\begin{array}{c}b_{1}(1235) \\
{[1229.5 \pm 3.2]} \\
761(0.2331)\end{array}$ & $\begin{array}{c}\pi_{2}(1670) \\
{[1672.4 \pm 3.2]} \\
1067(0.4143)\end{array}$ & & \\
\hline $\bar{s} u, \bar{s} d$ & $\begin{array}{c}K_{l}(1270) \\
{[1273 \pm 7]} \\
895.3(0.2947)\end{array}$ & $\begin{array}{c}K_{2}(1770) \\
{[1773 \pm 8]} \\
1166.8(0.4455)\end{array}$ & $\begin{array}{l}* K_{3}(2320) \\
{[2324 \pm 24]} \\
1386(0.87)\end{array}$ & $\begin{array}{c}* K_{4}(2500) \\
{[2490 \pm 20]} \\
1575.2(0.9297)\end{array}$ \\
\hline$\overline{s s}, \bar{d} d, \bar{u} u$ & $\begin{array}{c}h_{I}(1170)[1170 \pm 20] \\
h_{I}(1170)[1170 \pm 20] \\
900.8(0.1384) \\
(0.2774)\end{array}$ & $\begin{array}{c}\eta_{2}(1645)[1617 \pm 5] \\
\eta_{2}(1870)[1842 \pm 8] \\
1171(0.3109) \\
(0.5054)\end{array}$ & & \\
\hline $\bar{u} c, \bar{d} c$ & $\begin{array}{c}D_{1}(2420)[2422.2 \pm 1.8] \\
2009(0.4603)\end{array}$ & & & \\
\hline$\overline{S C}$ & $\begin{array}{c}D_{I}(2420)[2422.2 \pm 1.8] \\
2104(0.593)\end{array}$ & & & \\
\hline $\bar{C} C$ & $\begin{array}{c}h_{c}(1 P)[3526.21 \pm 0.25] \\
3058(0.7707)\end{array}$ & & & \\
\hline
\end{tabular}


Note that the predicted values in the second columns in Table 2 and Table 3 are the same for the same quark content. This is due to that (10) cannot distinguish between $s=J=1, I=0$ in Table 3 from $J=0, \mathrm{~s}=I=1$ in Table 4 . The differences are due to that the neglected $\Phi_{c 1}$ and $\Phi_{c o}$ are different; the latter also depends upon the angles in the intermediate $r$ region. The associated radial and orbital quantum numbers $n_{r}$ and $l$, meaningful at $r \rightarrow 0$ and $\infty$, are coupled in that region and may lead to a new pair of quantum numbers.

For orbitally excited triplet mesons he classification ([4] \$5.7.2) remains the same.

\section{Conclusions}

At very low energies, classical mechanics fails and has to be replaced by quantum mechanics, which can however go back to classical mechanics when the energy is sufficiently high. This is not true in the reverse direction. Similarly, QCD fails at low energies and has to be replaced by an appropriate low energy theory, here the SSI, which analogously can go over to QCD in a high energy region [8], Chapter 14 of [4]. Again, this is not true in the reverse direction.

The role of the nonlinear potential (2) and (5) needs be investigated in an attempt to remove the discrepancies given in the parentheses (...) in Tables 2-4.

The book [4] remains the same up to equation (3.2.19); but (3.2.20), $d_{m 2}=0$, needs be changed to $d_{m}=0$ which will lead to changes in rest of the book. Thus, the decay rate calculations in Chapters 6-8 need be revised.

\section{References}

[1] Burgess, C. and Moore, G. (2007) The Standard Model, A Primer. Cambridge University Press, Cambridge.

[2] Hoh, F.C. (1993) International Journal of Theoretical Physics, 32, 1111-1133. https://doi.org/10.1007/BF00671793

[3] Hoh, F.C. (1996) Journal of Physics G: Nuclear and Particle Physics, 22, 85. https://doi.org/10.1088/0954-3899/22/1/007

[4] Hoh, F.C. (2011) Scalar Strong Interaction Hadron Theory. Nova Science Publishers, Hauppauge. (2017) revised second edition, open access at https://www.novapublishers.com/catalog/product_info.php?products_id $=27069$

[5] Patrignani, C., et al. (Particle Data Group) (2016) Chinese Physics C, 40, 100001.

[6] Lichtenberg, D.B. (1987) International Journal of Modern Physics A, 2, 1669. https://doi.org/10.1142/S0217751X87000879

[7] Ludwig, W. and Falter, C. (1988) Symmetry in Physics. Springer.

[8] Hoh, F.C. (2013) Journal of Modern Physics, 4, 1171-1175. https://doi.org/10.4236/jmp.2013.49157 
Submit or recommend next manuscript to SCIRP and we will provide best service for you:

Accepting pre-submission inquiries through Email, Facebook, LinkedIn, Twitter, etc. A wide selection of journals (inclusive of 9 subjects, more than 200 journals)

Providing 24-hour high-quality service

User-friendly online submission system

Fair and swift peer-review system

Efficient typesetting and proofreading procedure

Display of the result of downloads and visits, as well as the number of cited articles Maximum dissemination of your research work

Submit your manuscript at: http://papersubmission.scirp.org/

Or contact jmp@scirp.org 